\title{
Vacuum-Assisted Generation and Control of Atomic Coherences at X-Ray Energies
}

\author{
Kilian P. Heeg, ${ }^{1}$ Hans-Christian Wille, ${ }^{2}$ Kai Schlage, ${ }^{2}$ Tatyana Guryeva, ${ }^{2}$ Daniel Schumacher, ${ }^{2}$ Ingo Uschmann, ${ }^{3,4}$ \\ Kai S. Schulze, ${ }^{4}$ Berit Marx, ${ }^{3}$ Tino Kämpfer, ${ }^{4}$ Gerhard G. Paulus, ${ }^{3,4}$ Ralf Röhlsberger, ${ }^{2}$ and Jörg Evers ${ }^{1}$ \\ ${ }^{1}$ Max-Planck-Institut für Kernphysik, Saupfercheckweg 1, 69117 Heidelberg, Germany \\ ${ }^{2}$ Deutsches Elektronen-Synchrotron DESY, Notkestraße 85, 22607 Hamburg, Germany \\ ${ }^{3}$ Institut für Optik und Quantenelektronik, Friedrich-Schiller-Universität Jena, Max-Wien-Platz, 1, 07743 Jena, Germany \\ ${ }^{4}$ Helmholtz-Institut Jena, Fröbelstieg 3, 07743 Jena, Germany
}

(Received 3 May 2013; published 14 August 2013)

\begin{abstract}
The control of light-matter interaction at the quantum level usually requires coherent laser fields. But already an exchange of virtual photons with the electromagnetic vacuum field alone can lead to quantum coherences, which subsequently suppress spontaneous emission. We demonstrate such spontaneously generated coherences (SGC) in a large ensemble of nuclei operating in the x-ray regime, resonantly coupled to a common cavity environment. The observed SGC originates from two fundamentally different mechanisms related to cooperative emission and magnetically controlled anisotropy of the cavity vacuum. This approach opens new perspectives for quantum control, quantum state engineering and simulation of quantum many-body physics in an essentially decoherence-free setting.
\end{abstract}

DOI: 10.1103/PhysRevLett.111.073601

PACS numbers: 42.50.Nn, 03.65.Yz, 42.50.Ct

Light-matter interaction at the quantum level is ubiquitous in a multitude of modern applications and in fundamental studies on the foundations of physics alike. Even the seemingly simple process of spontaneous emission (SE) of a quantum system (e.g., an atom) is surprisingly complex [1]. It arises from the exchange of virtual photons between the atom and the surrounding vacuum. SE is a major obstacle in quantum engineering, as it destroys coherence. But somewhat surprisingly, the energy exchange between atom and vacuum can also create coherences between different states of the atom, if emission and reabsorption of the virtual photon occur on different transitions within the atom. In turn, these so-called spontaneously generated coherences (SGC) [1-3] enable interference between different SE channels, such that unwanted SE can be modified or even suppressed. Therefore, SGC are a powerful resource in quantum engineering, and numerous fascinating applications have been suggested like lasing without inversion [4,5], enhancing nonlinear responses [6], quantum control of light propagation [7], quantum coherence in semiconductor-based devices [8,9], creation of entanglement [10], stabilization of coherence in quantum computation schemes [11,12], or increasing the efficiency of solar cells [13,14].

The archetype model system for SGC is a three-level system with two upper and one common lower state ( $V$ configuration) [1-3]. If SGC between upper states is established, the system can be trapped in the excited states despite its coupling to the environment. Usually, two major requirements on the level structure, naturally not met in atoms, hinder an implementation of SGC. First, the dipole moments of the transitions absorbing and emitting the virtual photon must be nonorthogonal. Second, the involved transition energies have to be near-degenerate on the level of the respective transition widths. Then, the different decay pathways become indistinguishable [15]. Observations in artificial quantum systems [8] can be interpreted in terms of SGC [16]. An alternative route to observing SGC are $\Lambda$-type atoms in which one excited state decays to multiple lower states. Here, one can erase the knowledge to which lower state the atom has decayed via an additional interaction [17-20]. Alternatively, SGC have recently been realized in an artificial $\Lambda$-type quantum system [21]. But all of these approaches have the drawback that the SE of the excited state cannot be suppressed in $\Lambda$-type setups, prohibiting full quantum control of SE, and therefore, most desirable applications.

Here, we report a direct demonstration and control of $V$-type SGC in a cavity containing a large ensemble of ${ }^{57} \mathrm{Fe}$ Mössbauer nuclei, probed with $\mathrm{x}$ rays in resonance with the nuclear transition energy of $14.4 \mathrm{keV}$. Embedding ensembles of ${ }^{57} \mathrm{Fe}$ atoms in planar cavities has recently facilitated extending quantum optical concepts into the regime of hard $\mathrm{x}$ rays $[22,23]$. For the observation of $\mathrm{SGC}$ we generalize this approach by capitalizing the magnetic hyperfine splitting of the Mössbauer line, resulting in up to six dipole-allowed transitions, see Fig. 1. This not only substantially enlarges the level space available for advanced applications, but also enables control of the system via the direction and magnitude of an applied external magnetic field. The spectral response of the system is probed via the reflectivity for near-resonant $x$ rays impinging in grazing incidence geometry on the cavity. The cavity is driven in the first guided mode where electronic scattering towards the direction of the detector vanishes [22].

We focus on three different orientations of the magnetic hyperfine field $\hat{\boldsymbol{B}}_{\boldsymbol{h} \boldsymbol{f}}$ with respect to the beam propagation direction $\hat{\boldsymbol{k}}_{\mathbf{0}}$, the layer surface normal $\boldsymbol{\pi}$, and $\boldsymbol{\sigma}=\left(\hat{\boldsymbol{k}}_{\mathbf{0}} \times \boldsymbol{\pi}\right)$ as shown in Fig. 1: (1) Faraday geometry: $\hat{\boldsymbol{B}}_{\boldsymbol{h f}} \| \hat{\boldsymbol{k}}_{\mathbf{0}}$, 


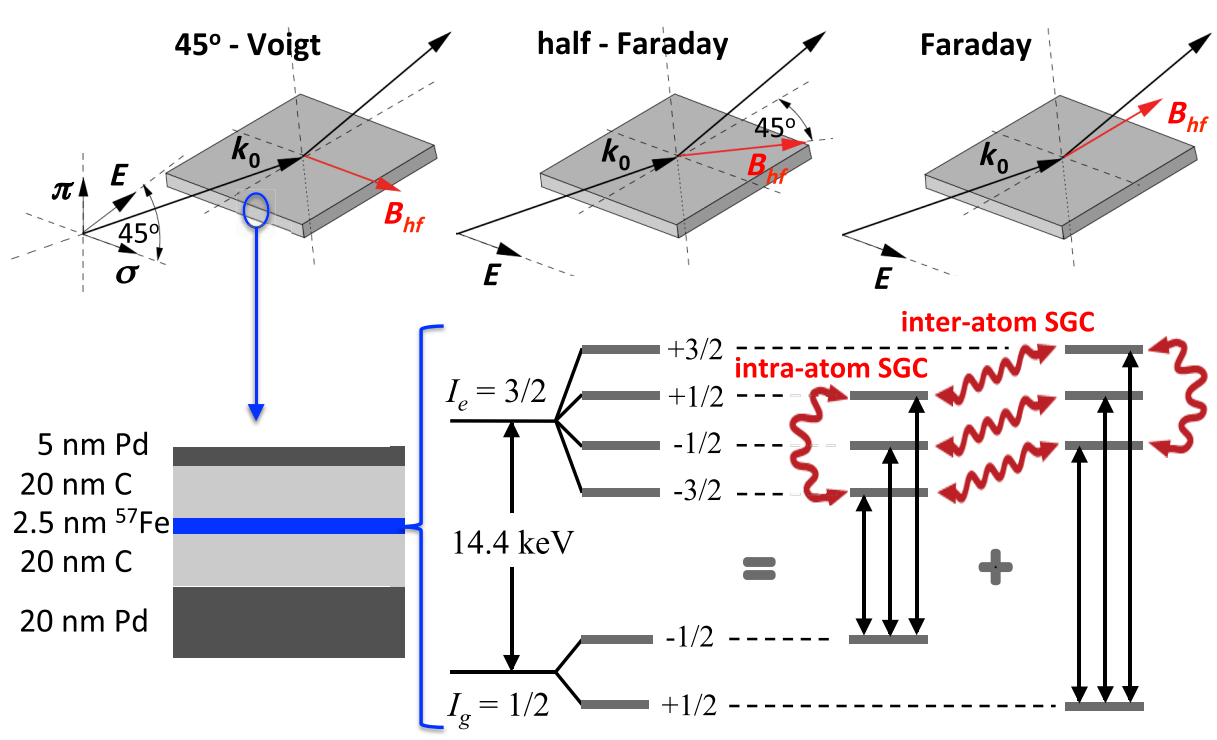

FIG. 1 (color online). Schematic representation of the sample and scattering geometries. The top panel shows three geometries for which the occurrence of SGC was investigated. Linearly polarized x rays are impinging under a grazing angle to evanescently couple into the first-order mode of the thin-film cavity (bottom left) and resonantly excite a thin layer of ${ }^{57} \mathrm{Fe}$ nuclei. The polarization plane of the incident and the detected x rays is defined by linear x-ray polarizer crystals before and behind the sample [31,35]. The magnetic hyperfine field $\boldsymbol{B}_{\boldsymbol{h} f}$ at the position of the nuclei defines the quantization axis. It can be aligned and controlled via a weak external magnetic field. $(\boldsymbol{\sigma}, \boldsymbol{\pi})$ denote the vectors of the linear polarization basis. The lower right panel shows the level scheme of the ${ }^{57} \mathrm{Fe}$ nucleus, subject to a magnetic hyperfine interaction, as well as a decomposition of the ensemble-cavity system into an effective level scheme. Initially, each nucleus is in one of its two ground states, such that the nuclei can be divided into two distinct groups. Within each of the two groups, the nuclei have a single ground state and three excited states accessed by the probing x-ray field. Cooperative effects lead to superradiant enhancement $\gamma_{S}$ of the natural decay rate $\gamma$, and to a cooperative Lamb shift $\Delta_{\mathrm{LS}}$. SGC occur due to the mode anisotropy and due to interactions between different nuclei, and are indicated by the curly arrows between the upper states.

(2) Half-Faraday geometry: $\hat{\boldsymbol{B}}_{\boldsymbol{h f}} \| \hat{\boldsymbol{k}}_{\mathbf{0}}+\boldsymbol{\sigma}$, and (3) $45^{\circ}$ Voigt geometry: $\hat{\boldsymbol{B}}_{\boldsymbol{h} \boldsymbol{f}} \| \boldsymbol{\sigma}$. We found that in all three cases, narrow spectral dips appear in the reflectivity, which in the case of the half-Faraday and the $45^{\circ}$-Voigt geometry lead to a vanishing signal at certain detunings. These signatures are clearly incompatible with the incoherent sum of different spectral lines, and thus point to interference that results from SGC as we will show in the following.

For the description of the observed reflected signal, conventionally, a self-consistent matrix formalism is applied [22-24] (for a review of this technique, see Supplemental Material [25]). Unfortunately, it does not provide a handle to interpret these signatures. To overcome this limitation, we have developed a full quantum optical theory for x-ray scattering from nuclei embedded in a cavity. It quantitatively agrees with previously used descriptions in the respective limits, but allows us to clearly identify, separate, and characterize all physically relevant processes contributing to the result, and, furthermore, provides the basis to naturally extend the modeling to nonclassical light fields and nonlinear light-nucleus interactions.

We model the combined system of the cavity and the nuclei as a single effective nucleus, but with level structure and properties crucially modified due to cooperativity and the cavity compared to a single bare nucleus. The excited level structure and the transition properties are determined by the externally controllable polarization and magnetization configuration, see Fig. 1. Initially, the nuclei are incoherently distributed over the two ground states. Including all possible polarization channels, each ground state can be coupled to at most three excited levels by the probing $\mathrm{x}$-ray field, such that our effective level scheme consists of one ground and six excited states, each one representing a possible transition in the ensemble of ${ }^{57} \mathrm{Fe}$ nuclei.

Next, we set up a master equation for the density matrix $\rho$ [1-3]. Using $\hbar=1$, the Hamiltonian is

$$
H=\sum_{i=1}^{n}\left(-\Delta+\Delta \omega_{i}\right) S_{i+} S_{i-}+\frac{1}{2} \Omega_{i} S_{i+}+\frac{1}{2} \Omega_{i}^{*} S_{i-},
$$

where $S_{i+}\left[S_{i-}\right]$ are the raising [lowering] operators of transition $i \in\{1, \ldots, 6\}, \Omega_{i}$ are the Rabi frequencies, $\Delta$ denotes the detuning between photon and transition energy at vanishing magnetization, and $\Delta \omega_{i}$ are Zeeman shifts. Moreover, we include

$$
H_{\mathrm{LS}}=\frac{\Delta_{\mathrm{LS}}}{2} \sum_{i=1}^{n} \sum_{j=1}^{n} G_{i} G_{j}\left(S_{i+} S_{j-}+\text { H.c. }\right),
$$

where $G_{n}$ is an effective Clebsch-Gordan coefficient also taking into account the geometrical setting. For $i=j$, this corresponds to the collective Lamb shift (LS) of 
level $i$. The $i \neq j$ terms describe a dynamical coupling between the excited levels. The master equation is $\partial_{t} \rho=$ $-i\left[H+H_{\mathrm{LS}}, \rho\right]+\mathcal{L}[\rho]$, where the first term models the coherent evolution. $\mathcal{L}[\rho]$ describes the incoherent dynamics, and has three parts. The first two characterize SE and superradiant decay (SR) from the excited levels with decay rates $\gamma$ and $\gamma_{S} \gg \gamma$, respectively, and are given by

$$
\mathcal{L}^{(\mathrm{SE}+\mathrm{SR})}[\rho]=-\frac{1}{2} \sum_{n=1}^{6}\left(\gamma+\gamma_{S} G_{n}^{2}\right)\left\{\left[S_{n+}, S_{n-} \rho\right]+\text { H.c. }\right\} .
$$

The third term evaluates to

$$
\mathcal{L}^{(\mathrm{SGC})}[\rho]=-\frac{\gamma_{S}}{2} \sum_{n, m \neq n} G_{n} G_{m}\left\{\left[S_{n+}, S_{m-} \rho\right]+\text { H.c. }\right\} .
$$

It involves spontaneous couplings between different transitions $n$ and $m$, which are the origin of SGC. Our analysis indicates that only this complete model leads to consistency with the experiment and previous theoretical approaches. More details on the model can be found in the Supplemental Material [25].

The steady state linear response of the system yields results analytically equivalent to that from the matrix formalism. But as our quantum optical approach has the distinct advantage of separating the different contributing physical mechanisms, we can easily quantify the effect of SGC on the spectra by artificially switching them off. In Fig. 2(a), we show the effect for the half-Faraday geometry, where indeed the SGC lead to the narrow dips in the spectra indicative of interference.

This result raises the question, why the SGC contributions are crucial in our setting, whereas they do not contribute, e.g., for atoms in free space. Interestingly, in our setup, SGC emerge due to two fundamentally different mechanisms. The first contribution, visualized in Fig. 2(c), occurs in single nuclei, and arises from a spatially anisotropic photonic density of states in the cavity experienced by the nuclei for certain parameter choices. To illustrate this, suppose a magnetization direction $\hat{\boldsymbol{B}}_{\boldsymbol{h f}} \| \boldsymbol{\pi}$. The twodimensional polarization space in the cavity transverse to the propagation direction $\hat{\boldsymbol{k}}_{\mathbf{0}}$ can be described by the orthonormal basis vectors $\boldsymbol{\pi}$ and $\boldsymbol{\sigma}$. In this configuration, the $\Delta m= \pm 1$ transitions have dipole moments proportional to $\boldsymbol{\sigma} \pm i \hat{\boldsymbol{k}}_{\mathbf{0}}$. Thus, circularly polarized photons only interact with the cavity mode polarized along $\boldsymbol{\sigma}$, but not with that along $\boldsymbol{\pi}$. As a result, the cavity appears to have a spatially anisotropic density of states, which leads to SGC as predicted theoretically in $[26,27]$. In contrast, in free space, both polarizations contribute, and the two (nonzero) SGC contributions of the two polarizations cancel each other. This mechanism can also be interpreted in terms of a "hidden metastable state" [28]. In our case, the anisotropic vacuum leads to the formation of a nuclear state which is metastable with respect to the cavity mode. Note, however, that this state is not metastable with respect

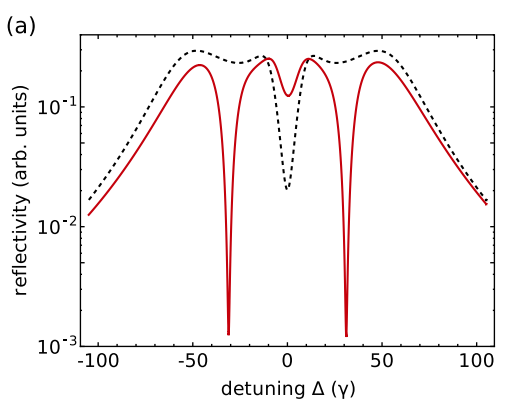

(b)

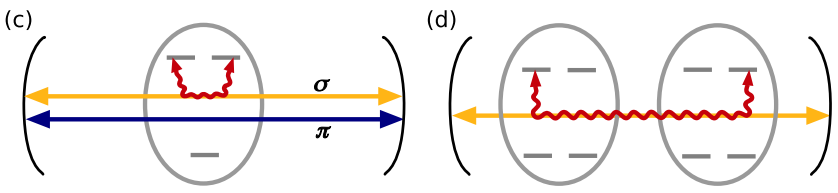

FIG. 2 (color online). Origin and effect of the spontaneously generated coherences. (a) Simulated spectra for the half-Faraday geometry obtained from the full quantum optical model. Solid line: expected result; dashed line: model calculation omitting the SGC contributions. The suppression of spontaneous emission at certain detunings due to the SGC is clearly visible. The superradiant decay rate enhancement and the SGC couplings are $\gamma_{S}=27 \gamma$, and the collective Lamb shift is $\Delta_{\mathrm{LS}}=1 \gamma$. (b) Effective single particle level scheme probed by the external field for the $45^{\circ}$-Voigt geometry. SGC are indicated by the curly arrows. (c) For certain orientations of $\hat{\boldsymbol{B}}_{\boldsymbol{h} f}$ relative to $\hat{\boldsymbol{k}}_{\mathbf{0}}$, the relevant nuclear transitions couple only to a single cavity polarization, giving rise to an anisotropic cavity vacuum and SGC between orthogonal transition dipoles. (d) In a collective effect photons are exchanged between different transitions in different nuclei. Probing the cavity as a whole results in effective SGC.

to the remaining free space vacuum modes, and, therefore, does not exist in free space. Finally, we note that even though being a single-nucleus effect, it is assisted by cooperativity, since superradiant line broadening larger than the energetic splitting of the two transitions renders them indistinguishable.

The second mechanism causing SGC is a collective effect involving multiple nuclei, see Fig. 2(d). Suppose, a photon is emitted by one nucleus with linear polarization on a $m_{e}=1 / 2 \rightarrow m_{g}=1 / 2$ transition. It can be reabsorbed in a different nucleus on the $m_{g}=-1 / 2 \rightarrow m_{e}=-1 / 2$ transition since the dipole moments are parallel. On this microscopic level, this constitutes an interaction between two different nuclei. The probe beam, however, does not resolve the dynamics of the individual nuclei, but probes the ensemble-cavity system as a whole. As a consequence, this exchange process inside the cavity appears as an effective coupling between different excited states within the level scheme of the single effective nucleus, see Fig. 2(b). In this sense, the complicated many-body dynamics of the ensemble of nuclei mediated by the cavity acts as a "quantum simulator" [29], which mimics a single effective quantum system with properties which go beyond those of each of the individual nuclei. Here, we specifically exploit this simulation technique to induce SGC in the 
effective level scheme observed by the $\mathrm{x}$-ray beam probing the total ensemble-cavity system.

To verify the SGC experimentally, we prepared a planar $\mathrm{x}$-ray cavity consisting of a $\mathrm{Pd}(5 \mathrm{~nm}) / \mathrm{C}(40 \mathrm{~nm}) / \mathrm{Pd}(20 \mathrm{~nm})$ layer system with the Pd layers acting as the mirrors and the $\mathrm{C}$ as guiding layer. A $2.5 \mathrm{~nm}$ thick ${ }^{57} \mathrm{Fe}$ layer was placed in the center of the carbon layer. In order to avoid perturbing nuclear hyperfine interactions at the ${ }^{57} \mathrm{Fe} / \mathrm{C}$ interface, the ${ }^{57} \mathrm{Fe}$ was sandwiched between two $0.6 \mathrm{~nm}$ layers of ${ }^{56} \mathrm{Fe}$, which in the present context has identical properties except for the resonance that we probe. In this environment, the Fe layer orders ferromagnetically and the magnetization can be aligned and controlled via a weak external field. The internal magnetic hyperfine field at the ${ }^{57} \mathrm{Fe}$ nuclei amounts to $33.3 \mathrm{~T}$ which results in a Zeeman splitting of the ground and excited state. Their respective splittings of adjacent states are $39.7 \gamma$ and $22.4 \gamma$ [30], where $\gamma=4.7 \mathrm{neV}$ is the natural line width of the Mössbauer transition.

The experiments were performed at the PETRA III synchrotron radiation source (DESY, Hamburg) employing the method of nuclear resonant scattering. This technique relies on the pulsed broadband excitation of nuclear levels followed by the time-resolved detection of the delayed photons. To determine the energy spectrum of the cavity reflectivity, we used a method similar to that reported in Ref. [22] (see Supplemental Material [25]). For the detection we employ two different approaches. First, an x-ray polarimetry setup was integrated into the experiment [31] in order to take full advantage of the six possible polarization-sensitive transitions resulting from the magnetic hyperfine splitting. The polarimeter consists of two $\mathrm{Si}$ (840) polarizer crystals in crossed setting with the sample in between, so that it ideally only transmits photons whose polarization has been rotated $(\boldsymbol{\sigma} \rightarrow \boldsymbol{\pi})$ upon the interaction with the nuclei. This way, nonresonant background photons are suppressed by almost 10 orders of magnitude. However, the polarimeter setup can act as an interferometer, in which the analyzer erases which-way information for different scattering channels for photons interacting with the sample. In particular, the central dip predicted for the $45^{\circ}$-Voigt geometry caused by SGC is superimposed with an interference structure induced by the detection setup if the analyzer is used. To clearly separate the effect of SGC, we omitted the analyzer in a second detection approach and recorded the spectrum for the $45^{\circ}$-Voigt geometry using a high resolution monochromator for the incident light to suppress the nonresonant background. This way, all interference structures can directly be attributed to SGC.

The measured as well as the calculated spectra obtained by the quantum optical model are shown in Fig. 3. Taking into account the detection technique, the numerical simulations reproduce the data very well. In particular, the deep interference minima due to SGC are clearly visible. In the case of the Faraday geometry it turned out that the spectrum can be explained only if, quite conceivably, a slight
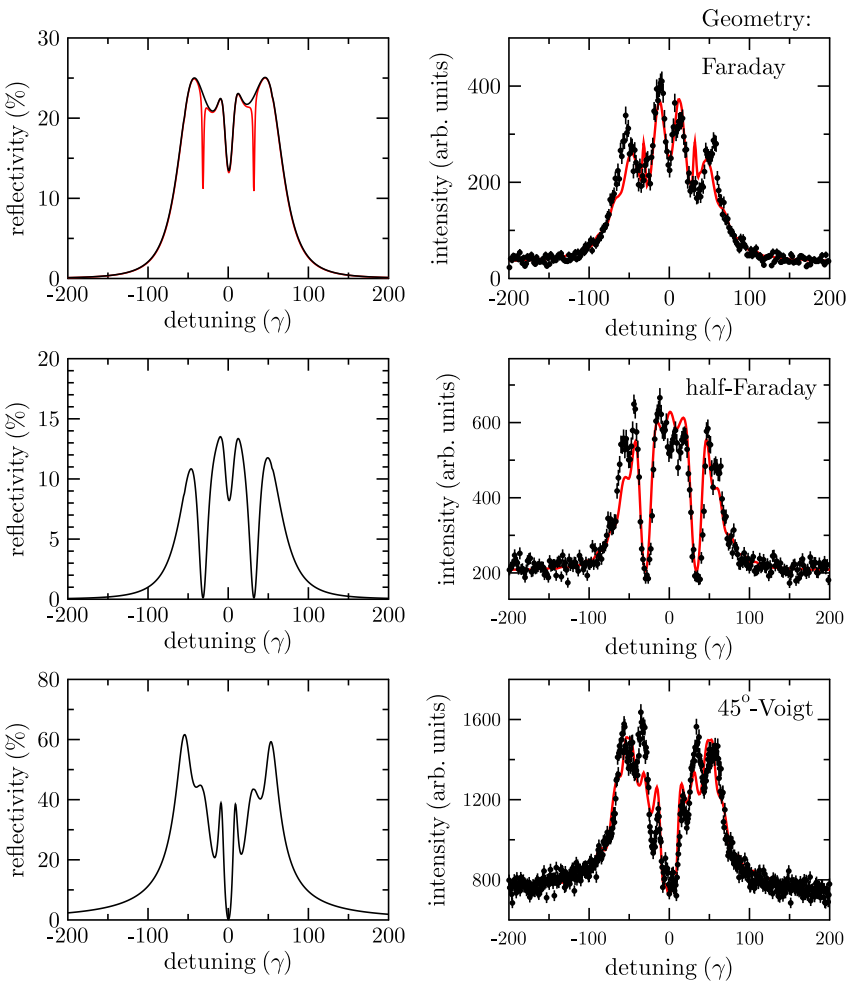

FIG. 3 (color online). Theoretical predictions and experimental results. The three rows show data for the three magnetization geometries introduced above. Left column: Theoretical predictions obtained from the quantum optical model. Right column: Experimental data and numerical simulations taking into account the scattering geometry, the measurement process, and the sample parameters. The additional red curve in the top left panel shows the result predicted with a small angular deviation used to model the experimental data in the top right panel. The good agreement between theory and experiment in all cases is clearly visible. The dips in the reflected intensity down to the background baseline for the half-Faraday and the $45^{\circ}$-Voigt geometry clearly indicate the presence of SGC in an essentially decoherence-free system.

misalignment of the internal magnetic field is assumed. As calculations indicate, this causes further minima already for small deviations from the exact Faraday geometry. Interestingly, we found that these minima are not present when switching off the SGC artificially. Thus, they are further evidence for SGC. The remaining difference between the quantum optical model and the data is mainly due to time-gating effects during the measurement process, uncertainties in the cavity parameters, and due to inhomogeneities in the magnetization orientation in the cavity, e.g., due to boundary effects between different layers.

The reduction of the reflected intensity at certain detunings can directly be traced back to the presence of nondecaying metastable excited states, formed due to SGC [32]. Therefore, our measurements amount to a direct observation of SGC between excited states, inducing a modified spontaneous decay. It should be noted that we observe near-perfect interference minima in the 
half-Faraday and $45^{\circ}$-Voigt geometries as the reflected intensities drop down to the baseline. In the language of quantum optics, this indicates that the system is essentially decoherence-free over the experimental time scales, as any perturbation would inevitably lead to loss of coherence, and, therefore, of a reduction of the interference contrast. The three cases in Fig. 3 were implemented with the same cavity, but with different directions of the applied magnetization, demonstrating the external control of the system properties.

Our results not only provide an avenue to the exploitation of SGC, but also demonstrate that genuinely new systems like high-grade noise free quantum optical level schemes can be engineered in the nuclear and the optical regime alike. Capitalizing the hyperfine splitting together with a suitable choice of the polarization and the magnetization enables us to realize continuously tunable and dynamically reconfigurable quantum optical level schemes in the hard x-ray regime. Thus, a single simple solid state target system can be manipulated dynamically and on demand to perform different tasks. The range of accessible level schemes becomes even richer if cavities involving multiple ensembles of resonant nuclei are used [23], possibly subject to individually differing magnetizations. Recently, a more general theoretical framework towards the exploitation of quantum and nonlinear effects was also established [33]. Future setups could involve dynamical control of the physical target structure [34]. It should be noted that our approach to realize SGC is not restricted to nuclear resonances, but can also be applied with atoms, ions, or artificial quantum systems properly placed in cavities.

We are grateful to R. Rüffer for the loan of the stainless steel analyzer foil. Moreover, we thank F. U. Dill and A. Scholl for help with the experimental setup. Finally, we acknowledge discussions with G.S. Agarwal and C. H. Keitel.

[1] G.S. Agarwal, in Quantum Optics, Springer Tracts in Modern Physics Vol. 70 (Springer, Berlin, 1974), pp. $1-128$.

[2] Z. Ficek and S. Swain, Quantum Interference and Coherence: Theory and Experiments, Springer Series in Optical Sciences Vol. 100 (Springer Science, New York, 2005).

[3] M. Kiffner, M. Macovei, J. Evers, and C. Keitel, in Progress in Optics (Elsevier Science, Burlington, 2010), Vol. 55, pp. 85-197.

[4] M. Fleischhauer, C. H. Keitel, M. O. Scully, C. Su, B. T. Ulrich, and S.-Y. Zhu, Phys. Rev. A 46, 1468 (1992).

[5] S. E. Harris, Phys. Rev. Lett. 62, 1033 (1989).

[6] Y. Niu and S. Gong, Phys. Rev. A 73, 053811 (2006).

[7] P. Zhou and S. Swain, Phys. Rev. Lett. 78, 832 (1997).

[8] H. Schmidt, K. L. Campman, A.C. Gossard, and A. Imamoglu, Appl. Phys. Lett. 70, 3455 (1997).
[9] J. Faist, F. Capasso, C. Sirtori, K. W. West, and L. N. Pfeiffer, Nature (London) 390, 589 (1997).

[10] Z.-h. Tang, G.-x. Li, and Z. Ficek, Phys. Rev. A 82, 063837 (2010).

[11] D. Kielpinski, V. Meyer, M. A. Rowe, C. A. Sackett, W. M. Itano, C. Monroe, and D. J. Wineland, Science 291, 1013 (2001).

[12] F. Verstraete, M. M. Wolf, and J. I. Cirac, Nat. Phys. 5, 633 (2009).

[13] M. O. Scully, Phys. Rev. Lett. 104, 207701 (2010).

[14] M. O. Scully, K. R. Chapin, K.E. Dorfman, M. B. Kim, and A. Svidzinsky, Proc. Natl. Acad. Sci. U.S.A. 108, 15097 (2011).

[15] S. E. Economou, R.-B. Liu, L. J. Sham, and D. G. Steel, Phys. Rev. B 71, 195327 (2005).

[16] M. Fleischhauer, C. Keitel, L. Narducci, M. Scully, S.-Y. Zhu, and M. Zubairy, Opt. Commun. 94, 599 (1992).

[17] A. Aspect, E. Arimondo, R. Kaiser, N. Vansteenkiste, and C. Cohen-Tannoudji, J. Opt. Soc. Am. B 6, 2112 (1989).

[18] M. Schubert, I. Siemers, R. Blatt, W. Neuhauser, and P. E. Toschek, Phys. Rev. A 52, 2994 (1995).

[19] B. Weber, H.P. Specht, T. Müller, J. Bochmann, M. Mücke, D. L. Moehring, and G. Rempe, Phys. Rev. Lett. 102, 030501 (2009).

[20] D. G. Norris, L. A. Orozco, P. Barberis-Blostein, and H. J. Carmichael, Phys. Rev. Lett. 105, 123602 (2010).

[21] M. V. Gurudev Dutt, J. Cheng, B. Li, X. Xu, X. Li, P. R. Berman, D. G. Steel, A.S. Bracker, D. Gammon, S. E. Economou, R.-B. Liu, and L. J. Sham, Phys. Rev. Lett. 94, 227403 (2005).

[22] R. Röhlsberger, K. Schlage, B. Sahoo, S. Couet, and R. Rüffer, Science 328, 1248 (2010).

[23] R. Röhlsberger, H.-C. Wille, K. Schlage, and B. Sahoo, Nature (London) 482, 199 (2012).

[24] R. Röhlsberger, J. Bansmann, V. Senz, K. L. Jonas, A. Bettac, K. H. Meiwes-Broer, and O. Leupold, Phys. Rev. B 67, 245412 (2003).

[25] See Supplemental Material at http://link.aps.org/ supplemental/10.1103/PhysRevLett.111.073601 for details on the detection method, a review of the matrix formalism, and a detailed description of the quantum optical model.

[26] P. Zhou and S. Swain, Opt. Commun. 179, 267 (2000).

[27] G. S. Agarwal, Phys. Rev. Lett. 84, 5500 (2000).

[28] P. R. Berman, Phys. Rev. A 58, 4886 (1998).

[29] J. I. Cirac and P. Zoller, Nat. Phys. 8, 264 (2012).

[30] J. Hannon and G. Trammell, Hyperfine Interact. 123-124, 127 (1999).

[31] B. Marx, I. Uschmann, S. Höfer, R. Lötzsch, O. Wehrhan, E. Förster, M. Kaluza, T. Stöhlker, H. Gies, C. Detlefs, T. Roth, J. Härtwig, and G. G. Paulus, Opt. Commun. 284, 915 (2011).

[32] S.-Y. Zhu, R. C.F. Chan, and C.P. Lee, Phys. Rev. A 52, 710 (1995).

[33] K. P. Heeg and J. Evers, arXiv:1305.4239.

[34] R. N. Shakhmuratov, F. Vagizov, and O. Kocharovskaya, Phys. Rev. A 84, 043820 (2011).

[35] T. S. Toellner, E. E. Alp, W. Sturhahn, T. M. Mooney, X. Zhang, M. Ando, Y. Yoda, and S. Kikuta, Appl. Phys. Lett. 67, 1993 (1995). 